\title{
Plantas medicinais no controle de urolitíase
}

CRUCES, I.L ${ }^{1 *}$; PATELLI, T.H.C ${ }^{2}$; TASHIMA, C.M. ${ }^{2}$; MELLO-PEIXOTO, E.C.T ${ }^{2}$

${ }^{1}$ Acadêmica do curso de Graduação em Medicina Veterinária da Universidade Estadual do Norte do Paraná (UENP/Bandeirantes). Email: ilobinha@gmail.com. ${ }^{2}$ Centro de Ciências Agrárias - Universidade Estadual do Norte do Paraná (UENP/Bandeirantes).

RESUMO: Plantas medicinais têm sido utilizadas mundialmente para o controle de urolitíases na medicina humana. A ocorrência desta afecção tem aumentado em humanos, ruminantes, cães e gatos. Nos animais, fatores patofisiológicos adquiridos, congênitos ou familiares podem estar envolvidos, havendo similaridade ao que se observa em seres humanos. Porém, apesar dos avanços nas pesquisas, o emprego de plantas medicinais na medicina veterinária, ainda é pouco utilizado. Dessa forma, objetivou-se apresentar uma revisão bibliográfica dos resultados do uso de plantas medicinais no controle de urolitíase. As plantas utilizadas são: Ammi visnaga, Bergenia ligulata, Cynodon dactylon, Herniaria hirsuta e Phyllanthus niruri. A atividade antilitogênica foi relacionada às alterações da composição iônica da urina, atividade diurética, inibição de cristalização e agregação de cristais. Destaca-se o uso de Phyllanthus niruri, pela eficácia na prevenção dos urólitos. Extratos de Phyllanthus niruri normalizaram níveis altos de cálcio urinário, desfavorecendo os estágios iniciais de formação dos urólitos. Adicionalmente, foram associados à elevação da filtração glomerular e excreção urinária de ácido úrico sugerindo utilização potencial não só como efeito lítico e preventivo das calculoses, mas também em pacientes hiperuricêmicos e portadores de insuficiência renal. A partir dos resultados relatados, pode-se concluir que os trabalhos apresentados pela literatura atual são capazes de comprovar os efeitos benéficos do uso de plantas medicinais no controle de urolitíase. Entretanto, ainda são escassos os estudos realizados em animais domésticos, predominando ensaios desenvolvidos em roedores.

Palavras-chave: ervas medicinais, Phyllanthus niruri, urina, urólitos.

ABSTRACT: Using medicinal plants in the control of urolithiasis. Medicinal plants have been used worldwide in the prevention of urolithiasis. The occurrence of this disease has increased in men, ruminants, dogs and cats. With regard to animals, acquired (congenital or hereditary) pathophysiological factors may be involved, a fact that bears similarity to what has been observed in humans. Yet, despite advances in research, the use of medicinal plants in veterinary medicine remains still underutilized. Thus, the purpose of this study is to conduct a literature review on the use of medicinal plants in the control of urolithiasis. The plants used are: Ammi visnaga, Bergenia ligulata, Cynodon dactylon, Herniaria hirsute and Phyllanthus niruri. Antilithogenic activity was related to changes in the ionic composition of urine, diuretic activity and inhibition of crystallization and aggregation of crystals. It is worth highlighting the use of Phyllanthus niruri due to its efficiency in the prevention of uroliths. Phyllanthus niruri extracts normalized high levels of urinary calcium, inhibiting the formation of uroliths in the initial stages. Additionally, they were associated with a higher glomerular filtration and higher urinary excretion of uric acid. These results suggest a potential use due to its lytic and preventive effect, as well as due to its application to hyperuricemic patients having renal insufficiency. We can conclude that the studies that pertain to the current literature can demonstrate the beneficial effects of using medicinal herbs to control urolithiasis. However, studies conducted in domestic animals remain scant, while tests developed in rodents still predominate.

Keywords: medicinal herbs, Phyllanthus niruri, urine, uroliths.

\section{INTRODUÇÃO}

A urolitíase representa importante preocupação devido à sua alta incidência. Nos
Estados Unidos, os gastos médicos excedem 1,5 bilhões anualmente (Kumaran \& Patki, 2011), 
com incidência correspondendo de 0,4 a $2 \%$. $\mathrm{Na}$ Alemanha relatou-se incidência de 0,5 a $1 \%$ (Vrabelova et al., 2011). Sua ocorrência tem aumentado em humanos, ruminantes, cães e gatos. Nos animais, fatores patofisiológicos adquiridos, congênitos ou familiares podem estar envolvidos (Osborne et al., 2008a), havendo similaridade ao que se observa em seres humanos (Robinson et al., 2008).

A urolitíase acomete qualquer órgão urinário, principalmente vesícula urinária em cães (Lulich \& Osborne, 2008). Em machos ocorrem próximo ao óstio peniano, na transição entre a uretra peniana e pélvica, ou no arco isquiático (MorishinFilho et al., 2010). A diminuição ou bloqueio do fluxo urinário pode resultar em crise urêmica, mudanças na estrutura do rim, ureter, e insuficiência renal, determinando uma das emergências mais comuns em medicina veterinária (Oyafuso, 2008).

A urina é uma solução supersaturada de sais que em certas condições precipitam formando cristais microscópicos. Esses possibilitam à formação de um núcleo, dando início ao desenvolvimento do urólito (Monferdini \& Oliveira, 2009; Woottisin et al., 2011; Yasir \& Waqar, 2011). O núcleo pode ser composto de minerais ou estruturas como pêlo, material de sutura, vegetal ou fragmentos de cateter urinário. A partir do núcleo, observa-se a lâmina externa, uma camada de precipitados que envolve o núcleo, e cristais de superfície (Koehler et al., 2008; Ulrich et al., 2008). De acordo com Oyafuso et al. (2010), o núcleo deve ser analisado separadamente, pois sua composição pode sugerir anormalidades subjacentes à formação do urólito.

O entendimento das características físicoquímicas da urina e farmacofisiologia do trato urinário são fundamentais para o controle da urolitíase. Fatores como pH urinário, raça, sexo, idade, infecção urinária, dieta, medicações, anormalidades anatômicas e metabólicas, aumentam o risco de precipitação (Oyafuso, 2008), e devem ser considerados para prevenção, diagnóstico precoce e controle de recidivas. Cães de pequeno porte apresentam maior predisposição devido ao pequeno volume urinário (Monferdini \& Oliveira, 2009). Cães Dálmatas são propensos ao desenvolvimento de cálculos de urato, devido à deficiência hereditária no metabolismo de acido úrico. Urólitos de estruvita foram correlacionados às infecções do trato urinário em cães, enquanto que em gatos relacionou-se ao excesso de minerais e à supersaturação da urina (Rogers et al., 2011). Urólitos de oxalato de cálcio (CaOx) são frequentes em animais de 8 a 12 anos, enquanto que animais jovens apresentaram mais frequentemente urólitos de estruvita, devido às infecções por bactérias produtoras de urease, as quais estão associadas à deficiência de defesa do trato urinário (Koehler et al., 2008).

$\mathrm{O} \mathrm{pH}$ alcalino é fator de risco para precipitação de fosfato de cálcio (Tiselius, 2011), já a acidificação promove dissolução, aumento de cálcio livre, e excreção urinária de íons cálcio e fosfato. Entretanto, o aumento de cálcio livre, por outro lado, pode favorecer a precipitação de $\mathrm{CaOx}$. Entre $\mathrm{pH}$ de 5.2 - 5.8 verifica-se precipitação de ácido úrico, entre 5.0-6.0 precipitam-se sais de oxalato, e em pH 7 observa-se precipitados de hidroxiapatita. Análises quantitativas de 350.803 urólitos caninos e 94.778 felinos foram realizadas no Minnesota Urolith Center, de 1981 a 2007. Verificou-se que 96\% dos urólitos corresponderam aos uratos, e estruvita, cistina, oxalato e fosfato de cálcio, representaram menos de 1\% (Osborne et al., 2008b).

Quanto à composição, os urólitos podem ser classificados como simples, com predomínio maior ou igual a $70 \%$ de único mineral. Os compostos apresentam minerais distintos em camadas diferentes, e os mistos apresentam camadas com diferentes tipos de minerais sem predominância (Ulrich et al., 2008). Oyafuso et al. (2010) avaliaram quantitativamente a composição mineral de 156 urólitos obtidos de cães, e verificaram ocorrência de $79,5 \%$ simples, $18 \%$ compostos e $2,5 \%$ mistos. Ao contrário dos urólitos simples, o tratamento dos compostos é complexo, dessa forma sua análise é essencial para o estabelecimento do tratamento adequado.

Protocolos de tratamento de urolitíase em cães são semelhantes aos dos seres humanos, sendo intervenção cirúrgica, modificação da dieta e tratamento farmacológico, os procedimentos mais adotados. Entretanto, a dieta pode não ser efetiva, ou ser rejeitada, no caso do animal doméstico. Além disso, nenhum tratamento disponível é isento de efeitos colaterais, sendo frequente as recidivas. Plantas medicinais têm sido utilizadas mundialmente na medicina humana, comprovando-se eficiência na prevenção de recidivas. Entretanto, a sua aplicação na medicina veterinária, ainda é limitada. Dessa forma, objetivou-se apresentar os principais resultados do uso de plantas medicinais no controle de urolitíase.

\section{Principais plantas medicinais com potencial uso no controle de urolitíase}

As plantas medicinais são popularmente utilizadas considerando principalmente seu efeito diurético. Entretanto, alteração do $\mathrm{pH}$ urinário também foi registrada (Gaybullaev \& Kariev, 2012; Kachchhi et al., 2012), e este achado é particularmente importante pelo fato de que o $\mathrm{pH}$ da urina é considerado um dos fatores mais importantes associados à litogênese. Embora não haja informações sobre o completo mecanismo 
de ação dessas plantas, relacionou-se além da ação diurética, ação antibiótica e alterações da composição da urina, como a desagregação de mucoproteínas pela presença de saponinas.

A Bergenia ligulata é utilizada no sul da Ásia, principalmente na Índia e no Paquistão. Bashir \& Gilani (2009) estudaram o extrato hidrometanólico de rizoma in vitro e in vivo, nas dosagens de 5 e $10 \mathrm{mg} /$ $\mathrm{kg}$ em ratos Wistar. Esses pesquisadores observaram efeito antioxidante, diurético e inibição da agregação de cristais de $\mathrm{CaOx}$, bem como formação de cristais em soluções metaestáveis. Efeitos sobre a inibição da nucleação, e do crescimento de cristais de fosfato de cálcio dihidratado, já haviam sido obersevados por Joshi et al. (2005), ao avaliarem Bergenia ligulata e Tribulus terrestris.

Rubus idaeus é amplamente distribuída nos países mediterrâneos. Ghalayini et al. (2011) avaliaram extrato aquoso a $11,5 \%$, por 12 dias, em ratos com hiperoxalúria induzida por etileno glicol. Esses pesquisadores verificaram redução de oxalato, cálcio e fósforo urinário, aumento da excreção de creatinina, e uma maior deposição de $\mathrm{CaOx}$ nos rins dos animais não tratados.

Herniaria hirsuta é comumente utilizada no tratamento de nefrolitíase. Atmani \& Khan (2000) verificaram inibição na agregação de cristais e formação de cristais de $\mathrm{CaOx}$ de menor tamanho. Utilizando $50 \mathrm{mg} / \mathrm{mL}$ por três semanas, em ratos portadores de nefrolitíase induzida por etileno glicol, foi verificado efeito diurético e preventivo contra cálculos de CaOx (Atmani et al. (2004a). Posteriormente, esses resultados também foram observados utilizando-se de 200 a $800 \mu \mathrm{g} / \mathrm{mL}$ (Atmani et al. (2004b). Após incubação por 30 minutos a 1 hora, foi observada uma diminuição progressiva da adesão de cristais, e uma total remoção dos cristais a partir de $400 \mu \mathrm{g} / \mathrm{mL}$. Os autores sugeriram a presença de substâncias capazes de revestir os cristais de oxalato, bloqueando a adesão destes às células.

$\mathrm{Na}$ Índia, a Moringa oleifera Lam. (Moringaceae) é utilizada por sua atividade antiurolítica. Karadi et al. (2006) utilizaram ratos Wistar hiperoxalúricos induzidos por etileno glicol. A administração diária de $200 \mathrm{mg} / \mathrm{kg}$, do extrato da raiz a $1 \%(\mathrm{~m} / \mathrm{v})$, por via oral, reduziu $\mathrm{CaOx}$ e o fosfato urinário, além dos níveis séricos de ácido úrico e creatinina.

A Cynodon dactylon é usada no Marrocos como diurético e no tratamento de cálculo renal. O extrato aquoso $(250 \mathrm{mg} / \mathrm{kg})$, administrado por duas semanas em ratos portadores de nefrolitíase induzida por etileno glicol, determinou redução de deposição de $\mathrm{CaOx}$ e cristalúria caracterizada por cristais de menor tamanho (Atmani et al., 2009).

Woottisin et al. (2011) avaliaram o efeito do extrato aquoso de Orthosiphon grandiflorus, Hibiscus sabdariffa e Phyllanthus amarus sobre cálculos de $\mathrm{CaOx}$, em ratos com hiperoxalúria induzida por ácido glicólico. Foram utilizadas $3,5 \mathrm{mg}$ diárias, por quatro semanas. Os extratos de Hibiscus sabdariffa e Phyllanthus amarus diminuíram a deposição de cristais nos rins. A diminuição da retenção de oxalato no rim e maior excreção na urina foram relacionadas à Hibiscus sabdariffa, enquanto que o efeito de Phyllanthus amarus pareceu depender mais do aumento de citrato urinário; que age diminuindo a concentração de íons de cálcio livre.

O chá dos frutos triturados de Ammi visnaga L. (Khella ) tem sido tradicionalmente usados no Egito. Abdel-Aal et al. (2009) observaram que o extrato de sementes $(1 \mu \mathrm{g}$ de semente seca/ $\mathrm{mL}$ de água) alterou cristais de $\mathrm{CaOx}$ monohidratado para forma dihidratada, e inibiu a nucleação de $\mathrm{CaOx}$ em $75 \%$ dos cristais. Vanachayangkul et al. (2010) observaram que os constituintes khellin e visnagin, reduziram a incidência de cristais de $\mathrm{CaOx}$ em ratos portadores de cálculo renal induzido por etileno glicol. Os extratos aquosos foram administrados por 14 dias, nas dosagens de 125, 250 e 500 mg/ kg por via oral, e propiciaram aumento da excreção de citrato e do $\mathrm{pH}$ urinário.

A Boerhaavia diffusa Linn (família Nyctaginaceae), usada na Índia e Brasil (erva tostão) para tratamento da urolitíase, foi avaliada por Pareta et al. (2011). Ratos com hiperoxalúria induzida por etileno glicol, foram tratados com extrato aquoso da raiz (100-200 mg/kg) por 28 dias. Observou-se a prevenção da poliúria, perda de peso, hiperoxalúria, distúrbios da função renal e inibição de deposição de $\mathrm{CaOx}$ nos túbulos renais. Complementarmente esses autores registraram inibição dos processos de nucleação e agregação, ao incubarem, a $37^{\circ} \mathrm{C}$ por 30 minutos, $2 \mathrm{~mL}$ de urina e $0,2 \mathrm{~mL}$ de extrato aquoso de Boerhaavia diffusa a 5, 10 e $20 \%$. Resultados semelhantes também foram registrados por Yasir \& Waqar (2011) ao avaliaram os extratos aquosos de Boerhavia diffusa e Bryophyllum pinnatum. As duas plantas reduziam os cristais e promoveram preferencialmente a formação de cristais de $\mathrm{CaOx}$ dihidratado em relação ao monohidratado.

Medicamentos formulados pela associação de plantas medicinais, também têm sido utilizados com êxito. Uma fórmula tradicional chinesa (Alisma orientalis, Polyporus umbellatus, Atractylodes macrocephala, Poria cocos e Cinnamomon cássia), denominada Wulingsan (WLS), foi associada à inibição da nucleação, cristalização e agregação do $\mathrm{CaOx}$., em ratos portadores de nefrocalcinose induzida por etileno glicol e tratados (375 e $1125 \mathrm{mg} /$ $\mathrm{kg})$ por quatro semanas (Chen et al., 2007). Tsai et al. (2008) verificaram uma diminuição de cristais de CaOx, utlizando $100 \mathrm{~g}$ de WLS: 
O extrato composto por Didymocarpus pedicellata com, Saxifraga lingulata, Rubia cordifolia, Cyperus scariosus, Achyranthes aspera, Onosma bracteatum, e Vernonia cineria (Cystone ${ }^{\circledR}$ ) é tradicionalmente usado na Índia. Esta formulação é vendida mundialmente pela Himalaya Health Care, e nos Estados Unidos, é comercializada como Uricare ${ }^{\circledR}$ (Kumaran \& Patki, 2011). Comprovou-se diminuição da supersaturação da urina e a expulsão de cálculos (Mohanty et al., 2010; Erickson et al., 2011). Karmakar \& Patki, (2010) realizaram metaanálise de 50 estudos clínicos envolvendo 3037 pacientes, nos quais os tratamentos variavam de duas semanas a dois anos. Exceto em pacientes pediátricos, o fitoterápico Cystone $®$ foi usado na dose de dois comprimidos, duas vezes ao dia. Registrou-se o desaparecimento de cálculos, alívio sintomático, aumento do volume urinário, redução dos constituintes formadores de urólitos, e leves efeitos colaterais como indigestão, irritação gástrica e flatulência. Demonstrou-se assim, uma eficiência no controle da urolitíase, especialmente de localização ureteral. Adicionalmente, verificouse uma redução na excreção urinária de oxalato, ácido úrico, cálcio, magnésio e fósforo, com significante aumento do volume urinário.

Os principais resultados da atividade antiurolítica de plantas medicinais, relatados pela literatura foram sumarizados na Tabela 1.

\section{Phyllanthus niruri: principais resultados no controle de urolitíases}

Devido ao seu uso difundido em todo Brasil (Marques, 2010), o presente estudo destaca as atividades da Phyllanthus niruri. Esta planta é tradicionalmente usada na medicina popular brasileira, sendo conhecida como "quebra pedra", "erva pombinha" e "arrebenta pedra" (Calixto et al., 1998). Pertencente as Euforbiáceas, este gênero compreende mais de 600 espécies (Mellinger, 2006). Outras espécies como Euphorbia prostrata, E. serpens, Phyllanthus tenellus, Desmodium incanum, Cunila microcephala e Heimia salicifolia também são conhecidas como quebra-pedra (Aita et al., 2009).

TABELA 1. Atividade antiurolítica de plantas medicinais, resultados apresentados pela literatura consultada.

\begin{tabular}{|c|c|c|}
\hline Planta Medicinal & Resultados (estudos in vitro / in vivo) & Referências \\
\hline $\begin{array}{l}\text { Ammi visnaga } \mathrm{L} \\
\text { (sementes) }\end{array}$ & $\begin{array}{l}\text { Alteração dos cristais de CaOx para forma dihidratada e inibição da nucleação } \\
\text { de CaOx em } 75 \% \text { dos cristais, e redução desses cristais em ratos portadores } \\
\text { de cálculo renal. }\end{array}$ & $\begin{array}{l}\text { Abdel-Aal et al. } \\
\text { (2009) }\end{array}$ \\
\hline $\begin{array}{l}\text { Ammi visnaga L. } \\
\text { ( sementes) }\end{array}$ & $\begin{array}{l}\text { Redução de cristais de } \mathrm{CaOx} \text {, redução de oxalato urinário, aumento da excreção } \\
\text { de citrato, alcalinização do pH urinário. }\end{array}$ & $\begin{array}{l}\text { Vanachayangkul } \\
\text { et al. (2010 2011) }\end{array}$ \\
\hline $\begin{array}{l}\text { Bergenia ligulata } \\
\text { (rizoma) }\end{array}$ & $\begin{array}{l}\text { Inibição da agregação de cristais de CaOx, efeito antioxidante, diurético, e } \\
\text { inibição de cristais (ratos Wistar). }\end{array}$ & $\begin{array}{l}\text { Bashir \& Gilani } \\
(2009)\end{array}$ \\
\hline $\begin{array}{l}\text { Bergenia ligulata } \\
\text { Tribulus terrestris } \\
\text { (raiz) }\end{array}$ & $\begin{array}{l}\text { Inibição da nucleação e do crescimento de cristais de fosfato de cálcio } \\
\text { dihidratado (experimentação in vitro utilizando urina artificial e humana). }\end{array}$ & $\begin{array}{l}\text { Joshi et al. } \\
(2005)\end{array}$ \\
\hline $\begin{array}{l}\text { Boerhaavia diffusa } \\
\text { Linn } \\
\text { ( raiz) }\end{array}$ & $\begin{array}{l}\text { Inibição de nucleação e agregação de cristais, prevenção de poliúria, perda } \\
\text { de peso, hiperoxalúria, distúrbios da função renal e inibição de deposição de } \\
\text { CaOx nos túbulos renais. }\end{array}$ & $\begin{array}{l}\text { Pareta et al. } \\
(2011)\end{array}$ \\
\hline $\begin{array}{l}\text { Boerhavia diffusa } \\
\text { e Bryophyllum } \\
\text { pinnatum (raiz) }\end{array}$ & $\begin{array}{l}\text { Redução dos cristais com promoção da forma de CaOx dihidratado em relação } \\
\text { ao monohidratado (experimentação in vitro: urina humana). }\end{array}$ & $\begin{array}{l}\text { Yasir \& Waqar } \\
(2011)\end{array}$ \\
\hline $\begin{array}{l}\text { Cynodon dactylon } \\
\text { (rizoma) }\end{array}$ & $\begin{array}{l}\text { Redução de deposição de } \mathrm{CaOx} \text { e cristalúria caracterizada por cristais de } \\
\text { menor tamanho. }\end{array}$ & $\begin{array}{l}\text { Atmani et al. } \\
(2009)\end{array}$ \\
\hline
\end{tabular}


TABELA 1. Atividade antiurolítica de plantas medicinais, resultados apresentados pela literatura consultada.

\begin{tabular}{|c|c|c|}
\hline Planta Medicinal & Resultados (estudos in vitro / in vivo) & Referências \\
\hline $\begin{array}{l}\text { Herniaria hirsuta } \\
\text { (planta toda) } \\
\text { Herniaria hirsuta } \\
\text { (planta toda) }\end{array}$ & $\begin{array}{l}\text { Efeito diurético e preventivo sobre cálculos de CaOx. Inibição de agregação e } \\
\text { formação de cristais de menor tamanho. } \\
\text { Diminuição da adesão de cristais e total remoção dos cristais a partir de } 400 \\
\mu \mathrm{g} / \mathrm{mL} \text {. Sugeriram presença de substâncias de revestimento inibindo a adesão } \\
\text { dos cristais às células renais. }\end{array}$ & $\begin{array}{l}\text { Atmani et al. } \\
\text { (2004a) } \\
\text { Atmani et al. } \\
(2004 b)\end{array}$ \\
\hline $\begin{array}{l}\text { Moringa oleifera Lam } \\
\text { (raiz) }\end{array}$ & $\begin{array}{l}\text { Reduziu o CaOx e fosfato urinário, além dos níveis séricos de ácido úrico e } \\
\text { creatinina (ratos Wistar hiperoxalúricos induzidos) }\end{array}$ & $\begin{array}{l}\text { Karadi et al. } \\
(2006)\end{array}$ \\
\hline $\begin{array}{l}\text { Orthosiphon } \\
\text { grandiflorus, } \\
\text { Hibiscus sabdariffa e } \\
\text { Phyllanthus amarus } \\
\text { (planta toda) }\end{array}$ & $\begin{array}{l}\text { Hibiscus sabdariffa e Phyllanthus amarus diminuíram a deposição de cristais } \\
\text { nos rins. A redução da retenção de oxalato no rim e maior excreção na urina } \\
\text { foram relacionadas à Hibiscus sabdariffa. Phyllanthus amarus influenciou o } \\
\text { aumento de citrato urinário, que diminui íons cálcio livre (ratos com hiperoxalúria } \\
\text { induzida). }\end{array}$ & $\begin{array}{l}\text { Woottisin et al. } \\
(2011)\end{array}$ \\
\hline $\begin{array}{l}\text { Rubus idaeus } \\
\text { (raiz) }\end{array}$ & $\begin{array}{l}\text { Efeito profilático sobre a formação de cálculos pela redução de oxalato, cálcio } \\
\text { e fósforo urinário. Aumento da excreção de creatinina (ratos com hiperoxalúria } \\
\text { induzida). }\end{array}$ & $\begin{array}{l}\text { Ghalayini et al. } \\
\text { (2011) }\end{array}$ \\
\hline $\begin{array}{l}\text { Wulingsan (WLS) } \\
\text { (planta toda) }\end{array}$ & $\begin{array}{l}\text { Inibição da nucleação, cristalização e agregação do CaOx (ratos portadores } \\
\text { de nefrocalcinose induzida). } \\
\text { Inibiu a deposição de cristais de } \mathrm{CaOx} \text { e a incidência de cálculos renais. }\end{array}$ & $\begin{array}{l}\text { Chen et al. } \\
(2007) \\
\text { Tsai et al. (2008) }\end{array}$ \\
\hline $\begin{array}{l}\text { Cystone } \AA \text { - na Índia } \\
\text { (ou Uricare }{ }^{\circledR} \text { nos EUA) }\end{array}$ & $\begin{array}{l}\text { Redução na excreção urinária de oxalato, ácido úrico, cálcio, magnésio e } \\
\text { fósforo, com aumento do volume urinário. Redução dos constituintes formadores } \\
\text { de urólitos, e de efeitos colaterais como indigestão, irritação gástrica e } \\
\text { flatulência. Diminuição da supersaturação da urina e a expulsão de cálculos } \\
\text { (Meta-análise de } 50 \text { estudos clínicos). }\end{array}$ & $\begin{array}{l}\text { Karamakar \& } \\
\text { Patki, (2010); } \\
\text { Kumaran \& Patki } \\
\text { (2011); Mohanty } \\
\text { et al. (2010); } \\
\text { Erickson et al. } \\
\text { (2011) }\end{array}$ \\
\hline
\end{tabular}

\footnotetext{
*Associação de 5 ervas: Alisma orientalis, Polyporus umbellatus, Atractylodes macrocephala, Poria cocos e Cinnamomon cássia).

* Extrato composto pelas plantas Didymocarpus pedicellata, Saxifraga lingulata, Rubia cordifolia, Cyperus scariosus, Achyranthes aspera, Onosma bracteatum, e Vernonia cineria.
}

Caracterizada como pequena erva de haste ereta, fina e ramosa, com 10 a $30 \mathrm{~cm}$ de altura, apresenta folhas ovais, flores amarelo-esverdeadas e frutos secos (Mellinger, 2006). Possui atividades anti-inflamatória, antifúngica, antiviral, antibacteriana, antioxidante, hepatoprotetora, hipoglicêmica, hipotensiva, analgésica e antilitogênica (Shanbhag et al., 2010). Na Índia é um importante fitoterápico comercializado (Sriarama et al., 2010), sendo exportado na forma de pó para formulações indicadas em problemas hepáticos (Kamble et al., 2008).

P. niruri tem sido avaliada para prevenção e dissolução de cálculos (Barros et al., 2003;
Micali et al., 2006; Shanbhag et al., 2010). Por apresentar propriedade uricosúrica, é de grande interesse no controle de hiperuricemia. Este fato é particularmente importante uma vez que $90 \%$ dos pacientes são subexcretores de ácido úrico (Murugaiyah \& Chan, 2009).

Mais de 50 compostos foram identificados no $P$. niruri, incluindo alcaloides, flavonoides, lignanas e triterpenos (Calixto et al., 1998). Os alcaloides presentes no gênero Phyllanthus apresentam atividade antiespasmódica, favorecendo o relaxamento do músculo liso (Boim et al., 2010). e a eliminação do cálculo urinário (Calixto et al., 1998). Os triterpenos inibem citotoxicidade induzida 
pelo $\mathrm{CaOx}$, além de reduzir deposição de cristais renais (Boim et al., 2010). Triterpenos pentacíclicos foram identificados como hepatoprotetores, hipolipidêmicos, antilitogênicos, anticancerígenos e antiinflamatórios (Barros et al., 2006). Malini et al. (2000) observaram que o lupeol, um dos triterpenos, reduziu a formação de cálculos pela diluição de promotores e proteção dos tecidos.

\section{vitro}

\section{Phyllanthus niruri: experimentação in}

Barros et al. (2003) verificaram inibição de $P$. niruri sobre a cristalização de $\mathrm{CaOx}$. A precipitação foi induzida por oxalato de sódio em urina não filtrada de ratos Wistar e humanos, na presença e ausência de extrato aquoso de $P$. niruri. $O P$. niruri não diminuiu o número de cristais, mas induziu redução do tamanho e agregação destes na urina humana. Segundo esses autores, esse efeito pode ter ocorrido devido à interferência nos estágios iniciais da formação de cálculos, representando assim uma forma adjuvante ao tratamento e prevenção da urolitíase.

Murugaiyah e Chan (2009) investigaram o efeito antihiperuricêmico da P. niruri; sobre a inibição da xantina. O extrato metanólico da planta inibiu moderadamente a xantina oxidase, apresentando concentração inibitória $\left(\mathrm{IC}_{50}\right)$ de $39,39 \mu \mathrm{g} / \mathrm{mL}$.

Ramsout et al. (2011) avaliaram o efeito do extrato aquoso à $5 \%(\mathrm{v} / \mathrm{v})$ sobre a cristalização de $\mathrm{CaOx}$ em urina sintética e real, incubadas por $2 \mathrm{~h}$ a $37^{\circ} \mathrm{C}$. A cristalização foi monitorada pelas taxas de nucleação, agregação e crescimento de cristais de $\mathrm{CaOx}$, avaliada por microscopia eletrônica de varredura. Na urina sintética ocorreu redução da taxa de crescimento com menores quantidades de cristais agregados, o que não foi observado em relação à urina real. Estes autores sugerem então cautela quando se extrapola os resultados de urina não real para condições clínicas.

\section{Phyllanthus niruri: experimentação in vivo}

Freitas et al. (2002) verificaram inibição na formação de cristais ao avaliarem urolitíase induzida por $\mathrm{CaOx}$ em ratos tratados com o $P$. niruri ( $1,25 \mathrm{mg} / \mathrm{mL}$ por 42 dias). Estes autores verificaram que não houve alteração na excreção de cálcio e oxalato. Dessa forma, sugeriram que o $P$. niruri não interferiu no transporte tubular destas substâncias, sendo a inibição da cristalização independente dos elementos litogênicos. O P. niruri também não interferiu na excreção de citrato e magnésio, indicando que o efeito anti-litogênico não foi mediado por esses inibidores.

O extrato metanólico da planta $P$. niruri, e seus constituintes lignanas, foram capazes de diminuir acido úrico plasmático de ratos hiperuricêmicos
(Murugaiyah \& Chan, 2006; Murugaiyah, 2008). Murugaiyah \& Chan (2009) demonstraram ainda um efeito anti-hiperuricêmico, ação uricosúrica pela inibição da xantina oxidase. Entretanto, essa inibição foi considerada relativamente fraca $(10 \mathrm{mg} / \mathrm{kg})$.

Barros et al. (2006) induziram a formação dos cálculos, por implantação cirúrgica de sementes de $\mathrm{CaOx}$ na vesícula urinária de ratos. Os animais foram distribuídos em grupos de acordo o início do tratamento: 20, 30 e 50 dias após implantação dos cálculos. Os cálculos formados após 50 ou 70 dias, foram pesados, fotografados por microscopia e analisados por difração de raios-X e microscopia eletrônica de varredura. Verificou-se um aumento de maciez e redução no número e peso dos cálculos em $75 \%$ e $65 \%$ (respectivamente), quando comparado aos animais não tratados. Entretanto, o tratamento não evitou o desenvolvimento de cálculos adicionais.

Os mecanismos do efeito antihiperuricêmico do extrato metanólico da $P$. niruri e das lignanas (fitalina, hipofitalina, filtetralina) isoladas, foram investigados em ratos hiperuricêmicos induzidos experimentalmente (Murugaiyah, 2008; Murugaiyah \& Chan, 2009). O P. niruri inibiu a xantina oxidase in vitro, e moderadamente in vivo, enquanto que as lignanas apresentaram fraca inibição. Entretanto, o tratamento intraperitoneal aumentou em 1,69 vezes a excreção de ácido úrico, comparado ao grupo controle, enquanto as lignanas aumentaram em 2,51 vezes a excreção na urina e 11 vezes sua concentração sérica. Esses autores concluíram que o efeito do extrato do $P$. niruri pode ser devido principalmente à ação uricosúrica e parcialmente pela inibição da xantina oxidase, enquanto que o efeito das lignanas foi atribuído à ação uricosúrica.

\section{Phyllanthus niruri: estudos clínicos}

O efeito de $P$ niruri $(450 \mathrm{mg}$ ) foi avaliado a cada 24 horas, por três meses, sobre os parâmetros bioquímicos do cálcio, ácido úrico, sódio e potássio no sangue, e cálcio, ácido úrico, citrato, magnésio, oxalato, sódio e potássio na urina de pacientes humanos portadores de cálculos de $\mathrm{CaOx}$. Estes pacientes apresentaram $38 \pm 8$ anos de idade, sendo 39 do sexo masculino e 30 do feminino. Os cálculos renais foram diadnosticados por ultrasonografia renal e raios- $X$ abdominal (Nishiura et al., 2004). Observou-se o controle da hipercalciúria e leve redução de magnésio na urina, mas a quantidade e tamanho dos cálculos não sofreram qualquer alteração, bem como os parâmetros urinários. Os pesquisadores concluíram que o tempo de estudo relativamente curto e o número pequeno de pacientes podem ter contribuído para os resultados negativos. Consideraram ainda, que a liofilização do extrato também pode ter influenciado negativamente. 
Micali et al. (2006), avaliaram 150 pacientes com cálculo renal de $25 \mathrm{~mm}$, compostos por CaOx. Todos foram submetidos de uma a três sessões de litotripsia, por meio de Dornier Lithotriptor $\mathrm{S}$. O extrato de $P$. niruri $(2 \mathrm{mg}$ por dia) foi administrado diariamente por pelo menos 3 meses, em $52 \%$ dos pacientes. Os demais foram considerados grupo controle. Os resultados radiográficos e ultrassonográficos não evidenciaram, até 180 dias, qualquer diferença no tamanho dos cálculos.

Quanto à toxicologia, Barros et al. (2006) relataram ausência de toxicidade aguda ou crônica, além de sugerir normalização dos níveis de cálcio e eliminação de urólitos em pessoas hipercalciúricas. Boim et al. (2010) não detectaram efeitos prejudiciais da Phyllanthus niruri sobre os sistemas renal, cardiológico e neurológico em seres humanos. Porém, apesar desses resultados, as plantas medicinais são passíveis de desenvolverem efeitos colaterais indesejáveis. Lin et al. (2012), verificaram tendência hemorrágica em ratos portadores de urolitíase, induzida por etilenoglicol, e tratados por Flos carthami, embora tenha sido demonstrada a inibição de deposição de sais de CaOx. Assim, o desenvolvimento de estudos clínicos determinando a eficácia da associação de plantas medicinais no controle de urolitíases (Saleem et al., 2012) é fator determinante para o avanço das pesquisas nesta área.

Em relação à medicina veterinária são escassos estudos relevantes desenvolvidos em animais domésticos. Trevisan et al. (2009), avaliaram tratamento fitoterápico experimental em ovino acometido por urolitíase obstrutiva pela presença cristais de estruvita (fosfato de amônio magnesiano). Esses autores registraram melhora clínica, com redução dos níveis séricos de creatinina, que foi mensurada durante os sete dias de tratamento. Entretanto, não especificaram as plantas constituintes do medicamento avaliado (DC 300). Um medicamento composto por plantas medicinais, dentre elas a Phyllanthus niruri, é produzido pela VetriScience ${ }^{\circledR}$ Laboratories de Vermont, uma divisão da Food Science Corporation - EUA (VetriScience, 2011). Este produto apresenta indicação contra urolitíases de cães e gatos. Entretanto, não foram encontrados estudos clínicos comprobatórios quanto à sua eficácia. Dessa forma, assim como observado em relação às demais plantas medicinais, estudos envolvendo a Phyllanthus niruri, são predominantemente desenvolvidos em roedores (Tabela 2).

\section{CONCLUSÕES}

Os trabalhos apresentados pela literatura atual são capazes de comprovar os efeitos benéficos do uso de plantas medicinais como terapia no controle de urolitíase. Destaca-se a eficácia da Phyllanthus niruri, na prevenção dos urólitos. Em relação aos estudos desenvolvidos em animais domésticos, estes ainda são escassos e predominantemente desenvolvidos em animais de laboratório como ratos e camundongos.

TABELA 2. Atividade antiurolítica de Phyllanthus niruri, resultados apresentados pela literatura consultada.

\begin{tabular}{|c|c|c|}
\hline $\begin{array}{l}\text { Parte da Planta } \\
\text { Phyllanthus niruri }\end{array}$ & Resultados (estudos in vitro / in vivo) & Referências \\
\hline Planta toda & $\begin{array}{l}\text { Reduziu tamanho e agregação de cristais na urina, sem alterar seu } \\
\text { número (In vitro: Urina não filtrada de ratos Wistar e humanos). }\end{array}$ & Barros et al. (2003) \\
\hline Planta toda & $\begin{array}{l}\text { Redução no número, peso e dureza dos cálculos, favorecendo } \\
\text { sua fragilidade e eliminação (ratos portadores de urolitíase vesical } \\
\text { induzida por etileno glicol). }\end{array}$ & Barros et al. (2006) \\
\hline Planta toda & $\begin{array}{l}\text { Inibição na agregação de } \mathrm{CaOx} \text { aos cristais pre-existentes e inibição } \\
\text { da formação de cristais (ratos portadores de urolitíase induzida por } \\
\mathrm{CaOx} \text { ) }\end{array}$ & Freitas et al. (2002) \\
\hline Folhas & $\begin{array}{l}\text { Inibição da xantina oxidase (ratos hiperuricosúricos induzidos por } \\
\text { oxonato de potássio e ácido úrico). }\end{array}$ & $\begin{array}{l}\text { Murugaiyah \& } \\
\text { Chan (2009) }\end{array}$ \\
\hline Planta toda & $\begin{array}{l}\text { A quantidade e o tamanho dos cálculos não sofreram qualquer } \\
\text { alteração (Humanos portadores de cálculos de cálcio). }\end{array}$ & $\begin{array}{l}\text { Nishiura et al. } \\
(2004) \text {; Micali et al. } \\
(2006)\end{array}$ \\
\hline Planta toda & $\begin{array}{l}\text { Redução de crescimento com menores quantidades de cristais } \\
\text { agregados (urina sintética e real) }\end{array}$ & $\begin{array}{l}\text { Ramsout et al. } \\
\text { (2011) }\end{array}$ \\
\hline
\end{tabular}

Rev. Bras. PI. Med., Campinas, v.15, n.4, supl.I, p.780-788, 2013. 


\section{REFERÊNCIA}

ABDEL-AAL, E.A.; DAOSUKHO, S.; EL-SHALL, H. Effect of supersaturation ratio and Khella extract on nucleation and morphology of kidney stones. Journal of Crystal Growth, v.311, p.2673-2681. 2009.

AITA, A.M. et al. Espécies medicinais comercializadas como "quebra-pedras" em Porto Alegre, Rio Grande do Sul, Brasil. Revista Brasileira de Farmacognosia. v. 19, n.2A, p.471-477, 2009.

ATMANI, F.; KHAN, S.R. Effect of an extract from Herniaria hirsuta on calcium oxalate crystallization in vitro. British Journal of Urology International, v.85, p.621-625, 2000.

ATMANI, F.; FARELL, G.; LIESKE, J.C. Effect of aqueous extract from Herniaria hirsuta L. on experimentally nephrolithiasic rats. Journal of Ethnopharmacology, v.95, p.87-93, 2004a.

ATMANI, F. et al. Extract from Herniaria hirsuta coats calcium oxalate monohydrate crystals and blocks their adhesion to renal epithelial cells. The Journal of Urology, v.172, p. 510-1514, 2004b.

ATMANI, F. et al. Cynodon dactylon extract as a preventive and curative agent in experimentally induced nephrolithiasis. Urological Research, v.37, p.75-82, 2009.

BARROS, M.E.; SCHOR, N.; BOIM, M.A. Effects of an aqueous extract from Phyllanthus niruri on calcium oxalate crystallization in vitro. Urological Research, v.30, p.374-379, 2003.

BARROS, M.E. et al. Effect of extract of Phyllanthus niruri on crystal deposition in experimental urolithiasis. Urological Research, v.34, p.351-357, 2006.

BASHIR, S.; GILANI, A.H. Antiurolithic effect of Bergenia ligulata rhizome: An explanation of the underlying mechanisms. Journal of Ethnopharmacology, v.122, p.106-116, 2009.

BOIM, M.A.; HEILBERG, I.P.; SCHOR, N. Phyllanthus niruri as a promising alternative treatment for nephrolithiasis. International Brazilian Journal of Urology, p.36, n.6, p.657-664, 2010.

CALIXTO, J.B. et al. A review of the plants of the genus phyllanthus: their chemistry, pharmacology, and therapeutic potential. Medical Research Reviews, v.18, n.4, p.225-258, 1998.

CHEN, Y.C. et al. Wu-Ling-San formula inhibits the crystallization of calcium oxalate in vitro. The American Journal of Chinese Medicine, v.35, p.533-541, 2007.

ERICKSON, S.B.; VRTISKAB, T.J.; LIESKE, J.C Effect of Cystone ${ }^{\circledR}$ on urinary composition and stone formation over a one year period. Phytomedicine, v.18, p.863867, 2011.

FREITAS, A.M.; SCHOR, N.; BOIM, M.A. The effect of Phyllanthus niruri on urinary inhibitors of calcium oxalate crystallization and other factors associated with renal stone formation. British Journal of Urology International, v.89, p.829-834, 2002.

GAYBULLAEV, A.; KARIEV, S. Phytotherapy of calcium urolithiasis with extracts of medicinal plants: Changes of diuresis, urine $\mathrm{pH}$ and crystalluria. Applied Technologies \& Innovations. v. 7, n. 2, p.59-66, 2012. GHALAYINI, I.F.; AI-GHAZO, M.A.; HARFEIL, N.A. Prophylaxis and therapeutic effects of raspberry (rubus idaeus) on renal stone formation in balb/c mice. International Brazilian Journal of Urology, v.37, n.2, p.259-267, 2011.

$\mathrm{KACHCHHI}$, N.R. et al. Evaluation of the antiurolithiatic activity of methanolic extract of celosia argentea roots in rats. International Journal of Phytopharmacology. v.3, n.3, p. 249-255, 2012.

KAMBLE, M.B.; DUMBRE, R.K.; RANGARI, V.D. Hepatoprotective studies of herbal formulations. International Journal of Green Pharmacy, v.2, p.147-151, 2008.

KARADI, R.V. et al. Effect of Moringa oleifera Lam. rootwood on ethylene glycol induced urolithiasis in rats. Journal of Ethnopharmacology, v.105, p.306-311, 2006.

KARMAKAR, D.; PATKI; P.S. Evaluation of efficacy and safety of herbal formulation Cystone in the management of Urolithiasis-meta analysis of 50 clinical studies. The Internet Journal of Alternative Medicine, v.8, p.1-18, 2010.

KOEHLER, L.A. et al. Canine Uroliths: Frequently Asked Questions and Their Answers. Veterinary Clinics Small Animal Pratice, v.39, p.161-181, 2008.

KUMARAN, M.G.S.; PATKI, P.S. Evaluation of an Ayurvedic formulation (Cystone), in urolithiasis: A double blind, placebo-controlled study. European Journal of Integrative Medicine, v.3, p.23-28, 2011.

JOSHI, V.S. et al. Inhibition of the growth of urinary calcium hydrogen phosphate dihydrate crystals with aqueous extracts of Tribulus terrestris and Bergenia ligulata. Urological Research, v.33, p.80-86, 2005.

LIN, W.C. et al. Protective effect of Flos carthami extract against ethylene glycol-induced urolithiasis in rats. Urology Research, v.40, p.655-661, 2012.

LULICH, J.P.; OSBORNE, C.A. Changing Paradigms in the Diagnosis of Urolithiasis. Veterinary Clinics Small Animal Pratice, n.39, p.79-91, 2008.

MALINI, M.M.; LENIN, M.; VARALAKSHMI, P. Protective effect of triterpenes on calcium oxalate crystal-induced peroxidative changes in experimental urolithiasis. Pharmacological Research, v.41, n.4, p.413-418, 2000.

MARQUES, L. C. Phyllanthus niruri (Quebra-Pedra) no Tratamento de Urolitíase: Proposta de Documentação para Registro Simplificado como Fitoterápico. Revista Fitos. v.5, n. 3, p.20-33, 2010.

MELLINGER, C.G. Caracterização estrutural e atividade biológica de carboidratos de Phyllanthus niruri (quebra-pedra). 2006. 138p. Tese (Doutorado - Área de concentração em Ciências Biológicas). Departamento de Bioquímica, UFPR, Curitiba.

MICALI, S. et al. Can Phyllanthus niruri affect the efficacy of extracorporeal shock wave lithotripsy for renal stones? A randomized, prospective, long-term study. The Journal of Urology, v.176, p.1020-1022, 2006.

MOHANTY, N.K.; NAYAK, R.L.; PATKI, P.S Safety and efficacy of Ayurvedic formulation Cystone in the management of ureteric calculi - a prospective randomized placebo controlled study. American Journal Pharmacology Toxic, v.5, n.2, p.58-64, 2010. MONFERDINI, R.P; OLIVEIRA, J. Manejo nutricional para cães e gatos com urolitíase - revisão bibliográfica. Acta Veterinaria Brasilica, v.3, n.1, p.1-4, 2009. 
MORISHIN FILHO, M.M.M. et al. Uretrolitíase em cães com quatro semanas de idade. Veterinária e Zootecnia, v.17, n.4, p.480-484, 2010.

MURUGAIYAH, V.; CHAN, K.L. Antihyperuricemic lignans from the leaves of Phyllanthus niruri. Planta Medica, v.72, p.1262-7, 2006.

MURUGAIYAH, V. Phytochemical, pharmacological and pharmacokinetic studies of phyllanthus niruri linn. lignans as potential antihyperuricemic agents. 2008. 54p. Tese (Doutorado - Filosofia). Universiti Sains Malaysia, Malaysia.

MURUGAIYAH, V.; CHAN, K.L. Mechanisms of antihyperuricemic effect of Phyllanthus niruri and its lignan constituents. Journal of Ethnopharmacology, v.124 p.233-239, 2009.

NISHIURA, J.L. et al. Phyllanthus niruri normalizes elevated urinary calcium levels in calcium stone forming (CSF) patients. Urological Research, v.32, p.362-366, 2004.

OSBORNE, C.A. et al. Melamine and Cyanuric Acid Induced Crystalluria, Uroliths, and Nephrotoxicity in Dogs and Cats. Veterinary Clinics Small Animal Pratice, v.39, p.1-14, 2008a.

OSBORNE, C.A. et al. Analysis of 451,891 canine uroliths, feline uroliths and feline urethral plugs from 1981 to 2007: perspectives from the Minnesota Urolith Center. Veterinary Clinics Small Animal Pratice, v.39, p.183197, 2008b.

OYAFUSO, M.K. Estudo retrospectivo e prospectivo da urolitíase em cães. Dissertação (Mestrado - área de concentração em Clínica Veterinária), 2008, 146p. Faculdade de Medicina Veterinária e Zootecnia, USP, São Paulo.

OYAFUSO, M.K. et al. Urolitíase em cães: avaliação quantitativa da composição mineral de 156 urólitos. Ciência Rural, v.40, n.1, p.102-108, 2010.

PARETA, S.K. et al. Prophylactic Role of Boerhaavia diffusa in Ethylene Glycol Induced Calcium Oxalate Urolithiasis. African Journal of Urology, v.17, n.2, p.28-36, 2011.

RAMSOUT, R.; RODGERS, A.; WEBBER, D Investigation of the effects of Phyllanthus nirurion in vitro calcium oxalate crystallization. European Urology Supplements, v.10, p.461-474, 2011.

ROBINSON, M.R. et al. Urolithiasis: Not Just a 2-Legged Animal Disease. The Journal of Urology, v.179, p.4652, 2008.

ROGERS, K.D. et al. Composition of uroliths in small domestic animals in the United Kingdom. The Veterinary Journal, v.188, p.228-230, 2011.
SALEEM, Q.E. et al. Clinical evaluation of herbal coded formulation urolith for treatment of urolithisis. Journal of Pharmacy and Pharmocology. v. 1, n.1, p. 5 9, 2012.

SHANBHAG, T. et al. Effect of Phyllanthus niruri. Linn on burn wound in rats. Asian Pacific Journal of Tropical Medicine, v.3, n.2, p.105-108, 2010.

SRIRAMA, R. et al. Assessing species admixtures in raw drug trade of Phyllanthus, a hepato-protective plant using molecular tools. Journal of Ethnopharmacology, v.130, p.208-215, 2010.

TISELIUS, H-G. A hypothesis of calcium stone formation: an interpretation of stone research during the past decades. Urological Research, v.39, n.4, p.231-243, 2011.

TSAI, C-H. et al. A traditional Chinese herbal antilithic formula, Wulingsan, effctively prevents the renal deposition of calcium oxalate crystal in ethylene glycolfed rats. Urological Research, v.36, p.17-24, 2008.

TREVISAN, L.F.A., et al. Tratamento fitoterápico experimental em ovino acometido por urolitíase obstrutiva. Agropecuária Técnica, v. 30, n. 2, 2009.

ULRICH, L.K. et al. Changing paradigms in the frequency and management of canine compound uroliths. Veterinary Clinics Small Animal Pratice, v.39, p.4153, 2008.

VANACHAYANGKUL, P. et al. An aqueous extract of Ammi visnaga fruits and its constituents khellin and visnagin prevent cell damage caused by oxalate in renal epithelial cells. Phytomedicine, v.17, p.653-658, 2010.

VANACHAYANGKUL, P. et al. Prevention of renal crystal deposition by an extract of Ammi visnaga $\mathrm{L}$. and its constituents khellin and visnagin in hyperoxaluric rats. Urological Research, v.39, p.189-195, 2011.

VETRISCIENCE LABORATORIES. UT STRENGTH STAT- A urinary tract support formula for dogs, 2011. Disponível em: <http://www.vetriscience.com/ ut-strength-dogs.php>. Acesso em: 24 set. 2011, 21:15.

VRABELOVA, D. et al. Analysis of 2735 canine uroliths in Spain and Portugal. A retrospective study: 20042006. Research in Veterinary Science, v.91, n.2, p.208-211, 2011.

WOOTTISIN, S. et al. Effects of Orthosiphon grandiflorus, Hibiscus sabdariffa and Phyllanthus amarus extracts on risk factors for urinary calcium oxalate stones in rats. The Journal of Urology, v.185, p.323-328, 2011.

YASIR, F.; WAQAR, M.A. Effect of indigenous plant extracts on calcium oxalate crystallization having a role in urolithiasis. Urological Research, v.39, n.5, p.345-350, 2011. 